\title{
A DIOPHANTINE EQUATION
}

\author{
by J. W. S. CASSELS
}

To Robert Rankin on the occasion of his 70 th birthday

0. I was recently challenged to find all the cases when the sum of three consecutive integral cubes is a square; that is to find all integral solutions $x, y$ of

$$
\begin{aligned}
y^{2} & =(x-1)^{3}+x^{3}+(x+1)^{3} \\
& =3 x\left(x^{2}+2\right) .
\end{aligned}
$$

This is an example of a curve of genus 1 . There is an effective procedure for finding all integral points on a given curve of genus 1 ([1, Theorem 4.2], [2]): that is, it can be guaranteed to find all the integral points and to show that no others exist with a finite amount of work. Unlike some effective procedures, which have only logical interest, this one can actually be carried out in practice, at least with the aid of a computer $([3],[5])$. There are, however, older methods for dealing with problems of this kind which, while not effective, very often lead more easily to a complete set of solutions (and a proof that it is complete). I solve the problem here by a technique introduced in [4]. It requires only the elementary theory of algebraic number-fields. The motivation is $p$-adic, but it is simpler not to introduce $p$-adic theory overtly.

There is a discussion of the problem in [6].

THEOREM 0.1. The only solutions of $(0.1)$ in integers are $x=0,1,2,24$.

We note that the greatest common factor of $x$ and $x^{2}+2$ is either 1 or 2 . Hence on considering the factorization of $x$ and $x^{2}+2$ in (0.1) there are integers $u, v$ such that one of the following holds:

$$
\begin{array}{ll}
x=3 u^{2}, & x^{2}+2=v^{2} \\
x=u^{2}, & x^{2}+2=3 v^{2} \\
x=2 u^{2}, & x^{2}+2=6 v^{2} \\
x=6 u^{2}, & x^{2}+2=2 v^{2}
\end{array}
$$

If $(0.2)$ holds, then

$$
9 u^{4}+2=v^{2}
$$

which is impossible modulo 3 . We treat the remaining equations $(0.3),(0.4),(0.5)$ in separate sections.

1. Here we deal with (0.3).

LEMMA 1.1. The only integral solution of

is $x=1$.

$$
x=u^{2}, \quad x^{2}+2=3 v^{2}
$$

Glasgow Math. J. 27 (1985) 11-18. 
Clearly $3 \nmid u$ so that $x=3 z-2$ for some integer $z$, and

$$
z^{2}+2(z-1)^{2}=v^{2}
$$

i.e.

$$
(v+z)(v-z)=2(z-1)^{2} .
$$

Any common prime divisor $p$ of $v+z, v-z$ divides their difference $2 z$ and it also divides $z-1$; so $p=2$. As $z, v$ are clearly both odd, there are integers $l, m$ such that one of the two following holds:

$$
\begin{array}{lll}
v+z=4 l^{2}, & v-z=2 m^{2}, & z-1=2 l m \\
v+z=2 l^{2}, & v-z=4 m^{2}, & z-1=2 l m .
\end{array}
$$

If (1.4) holds then

$$
1=2 l^{2}-m^{2}-2 l m=3 l^{2}-(l+m)^{2},
$$

which is impossible modulo 3 . Hence (1.5) holds, and so

and

$$
u^{2}=3 z-2=l^{2}+4 l m-2 m^{2}
$$

$$
1=l^{2}-2 l m-2 m^{2} \text {. }
$$

We now introduce $\gamma$, where

Then by (1.6), (1.7) we have

$$
\gamma^{2}=-2
$$

where

$$
\begin{aligned}
-\gamma & =u^{2}-(1+\gamma)(l-\gamma m)^{2} \\
& =u^{2}-(1+\gamma) \lambda^{2}
\end{aligned}
$$

$$
\lambda=l-\gamma m \text {. }
$$

We now work in the field $\mathbf{Q}(\gamma, \delta)$, where

so that (1.9) can be written

$$
\delta^{2}=1+\gamma
$$

$$
\operatorname{Norm}(u+\lambda \delta)=-\gamma
$$

where the Norm is taken from $\mathbf{Q}(\gamma, \delta)$ to $\mathbf{Q}(\gamma)$.

It is readily verified that 2 is completely ramified in $\mathbf{Q}(\gamma, \delta)$. There is thus a unique extension ||$_{2}$ of the 2-adic valuation to $\mathbf{Q}(\gamma, \delta)$ and

$$
|\gamma|_{2}=2^{-1 / 2}, \quad|\delta-1|_{2}=2^{-1 / 4} \text {. }
$$

It readily follows that $1, \gamma, \delta, \gamma \delta$ is a basis for the integers of $\mathbf{Q}(\gamma, \delta)$.

Solutions of (1.12) are clearly given by $u= \pm 1, \lambda= \pm 1$. We must show that these are the only solutions with $u \in \mathbf{Z}, \lambda \in \mathbf{Z}[\gamma]$. In any case,

$$
u+\lambda \delta=(1+\delta) \mu
$$


where $\mu$ is an integer (because of the complete ramification of 2), and so $\mu$ is a unit. [Note that this argument does not require a knowledge of the class-number of $\mathbf{Q}(\gamma, \delta)$.] Further,

where

$$
(1+\delta)^{2}=\gamma \eta
$$

$$
\eta=1-\gamma-\gamma \delta
$$

is a unit. Since $\operatorname{Norm}(1+\delta)=1-\delta^{2}=-\gamma$, it follows that

$$
(1+\delta) /(1-\delta)=-\eta \text {. }
$$

It is easy to verify that $\eta$ is a fundamental unit.

From all this it follows that

$$
u+\lambda \delta= \pm(1 \pm \delta) \eta^{2 n}
$$

for some $n \in \mathbf{Z}$ and some choices of signs. We have

where

$$
\eta^{ \pm 2}=1+\theta,
$$

$$
\theta=-4-4 \gamma \mp(4+2 \gamma) \delta .
$$

Suppose, if possible, that $n \neq 0$. Let $2^{r}$ be the highest power of 2 dividing $n$ and put $N=|n|$. Then

$$
\eta^{2 n}=(1+\theta)^{N}=1+N \theta+\sum_{2}^{N} T_{m}
$$

where

$$
T_{m}=\frac{N(N-1) \ldots(N-m+1)}{m !} \theta^{m} .
$$

Here $2^{[3 m / 2]}\left|\theta^{m}, 2^{r}\right| N$ and $2^{m} \nmid m !$. Hence

It follows that

$$
T_{m} \equiv 0 \quad\left(2^{r+2}\right) .
$$

$$
\begin{aligned}
\eta^{2 n} & \equiv 1+N \boldsymbol{\theta} \quad\left(2^{r+2}\right) \\
& \equiv 1+2^{r+1} \gamma \delta \quad\left(2^{r+2}\right) .
\end{aligned}
$$

Hence

$$
(1 \pm \delta) \eta^{2 n} \equiv 1+2^{r+1} \gamma+\left( \pm 1+2^{r+1} \gamma\right) \delta \quad\left(2^{r+2}\right) .
$$

In particular, the coefficient of $\gamma$ is non-zero, which contradicts (1.18).

2. Here we deal with (0.4).

LEMMA 2.1. The only integral solution of

is $x=2$.

$$
x=2 u^{2}, \quad x^{2}+2=6 v^{2}
$$

Here

$$
x=6 z+2
$$


for some $z \in \mathbf{Z}$, and so

that is

$$
2 z^{2}+(2 z+1)^{2}=v^{2}
$$

$$
\{v+(2 z+1)\}\{v-(2 z+1)\}=2 z^{2} .
$$

There are thus integers $l, m$ such that one of the two following holds:

$$
\begin{array}{lll}
v+2 z+1=4 l^{2}, & v-2 z-1=2 m^{2}, & z=2 l m ; \\
v+2 z+1=2 l^{2}, & v-2 z-1=4 m^{2}, & z=2 l m .
\end{array}
$$

If (2.5) holds, we have

$$
1=2 l^{2}-4 l m-m^{2}=6 l^{2}-(2 l+m)^{2},
$$

which is impossible modulo 3 .

Hence (2.6) holds, and

$$
\begin{aligned}
1 & =l^{2}-4 l m-2 m^{2}, \\
u^{2} & =l^{2}+2 l m-2 m^{2} .
\end{aligned}
$$

As in the preceding section, we define $\gamma$ by

Put

$$
\gamma^{2}=-2
$$

so that

$$
\lambda=l-\gamma m,
$$

$$
\begin{aligned}
1 & =-\gamma u^{2}+(1+\gamma) \lambda^{2} \\
& =\{u+(1+\gamma) \mu\}^{2}+(2-\gamma) \mu^{2},
\end{aligned}
$$

where

$$
\mu=\lambda-u \text {. }
$$

Clearly, solutions of (2.11) are given by $u= \pm 1, \lambda= \pm 1$. We shall show that these are the only solutions with $u \in \mathbf{Z}, \lambda \in \mathbf{Z}[\gamma]$.

The argument is similar to that in the previous section. We define $\delta$ now by

$$
\delta^{2}=-2+\gamma
$$

There is a unique extension ||$_{2}$ of the 2 -adic valuation and $|\gamma|_{2}=2^{-1 / 2},|\delta|_{2}=2^{-1 / 4}$. Hence $1, \gamma, \delta, \gamma \delta$ is a basis for the integers of $\mathbf{Q}(\gamma, \delta)$.

On putting $u=-1, \lambda=1$ in (2.11) we see that

$$
\eta=1+2 \gamma+2 \delta
$$

is a unit, and it is easy to check that it is fundamental. Then either

or

$$
\begin{aligned}
& u+(1+\gamma) \mu+\mu \delta= \pm \eta^{2 n} \\
& u+(1+\gamma) \mu+\mu \delta= \pm \eta^{1+2 n}
\end{aligned}
$$

for some $n \in \mathbf{Z}$. The proof now follows much as for Lemma 1.1 on noting that

$$
\eta^{\star 2}=1+\theta
$$


where

$$
\theta=-16+8 \gamma \pm(4+8 \gamma) \delta
$$

If $2^{r} \| n$ and $N=|n|$ we have

$$
\begin{array}{rlrl}
\eta^{2 n} & \equiv 1+N \theta \quad\left(2^{r+3}\right) \\
& \equiv 1+2^{r+2} \delta & \left(2^{r+3}\right) .
\end{array}
$$

This is incompatible with (2.15). Further,

which similarly contradicts $(2.16)$.

$$
\eta^{1+2 n} \equiv 1+2 \gamma+\left(2+2^{r+2}\right) \delta \quad\left(2^{r+3}\right)
$$

3. We now conclude the proof of Theorem 0.1 by dealing with $(0.5)$.

LEMMA 3.1. The only solutions in integers of

have $x=0$ or $x=24$.

$$
x=6 u^{2}, \quad x^{2}+2=2 v^{2}
$$

Clearly $v$ is odd. We have

$$
(v+1)(v-1)=\frac{1}{2} x^{2}=18 u^{4}
$$

and so there are integers $l, m$ such that one of the following holds:

$$
\begin{array}{ll}
v+1=144 l^{4}, & v-1=2 m^{4} \\
v+1=16 l^{4}, & v-1=18 m^{4} \\
v+1=2 l^{4}, & v-1=144 m^{4} \\
v+1=18 l^{4}, & v-1=16 m^{4} .
\end{array}
$$

On eliminating $v$, these give respectively:

$$
\begin{aligned}
& 72 l^{4}-m^{4}=1 ; \\
& 8 l^{4}-9 m^{4}=1 ; \\
& l^{4}-72 m^{4}=1 ; \\
& 9 l^{4}-8 m^{4}=1 .
\end{aligned}
$$

Here (3.7) and (3.8) are both impossible modulo 3. The only solutions of (3.9) have $m=0$, as follows from the next lemma.

LEMMA 3.2. All solutions in integers of

have $n=0$.

$$
l^{4}-2 n^{2}=1
$$

The proof is simple. First, $\left(l^{2}+1\right)\left(l^{2}-1\right)=2 n^{2}$. Here $l$ is odd, $l^{2}+1 \equiv 2(\bmod 4)$, and so $l^{2}+1=2 r^{2}, l^{2}-1=s^{2}$ for integers $r$, s. But then $(l+s)(l-s)=1$, so that $l+s= \pm 1$, $l-s= \pm 1$ and we are done. 
We note in passing that Lemma 3.2 implies the theorem of Skolem [9] which is reproduced on p. 207 of $[8]$.

There remains (3.10). We shall prove the following.

LEMMA 3.3. All integral solutions of (3.10) have $l^{2}=m^{2}=1$.

We have

Since

$$
\left(3 l^{2}+1\right)\left(3 l^{2}-1\right)=8 m^{4}
$$

$$
3 l^{2}-1 \equiv 2(\bmod 4)
$$

there are integers $r, s$ such that

$$
3 l^{2}+1=4 r^{4} ; \quad 3 l^{2}-1=2 s^{4},
$$

and so

$$
2 r^{4}-s^{4}=1
$$

Here one can invoke the deep theorem of Ljunggren [7] that the only positive solutions of $2 x^{4}-y^{2}=1$ are $(1,1)$ and $(13,239)$. Alternatively, we can proceed as follows.

LEMMA 3.4. All integral solutions of

have $s^{2}=t^{2}=1$.

$$
2 t^{2}-s^{4}=1
$$

Without loss of generality $t \geqslant 0$. We have

where

$$
\left(1+2 t+s^{2}\right)\left(1-2 t-s^{2}\right)=-2\left(t+s^{2}\right)^{2}
$$

$$
\begin{aligned}
& 1+2 t+s^{2}>0, \\
& 1-2 t-s^{2} \equiv 2(\bmod 4) .
\end{aligned}
$$

Hence there are integers $a, b$ such that

$$
\begin{aligned}
1+2 t+s^{2} & =4 a^{2} \\
1-2 t-s^{2} & =-2 b^{2} \\
t+s^{2} & =2 a b
\end{aligned}
$$

and so

$$
\begin{gathered}
2 a^{2}-b^{2}=1, \\
-2 a^{2}+4 a b-b^{2}=s^{2} .
\end{gathered}
$$

We operate now in $\mathbf{Q}^{(i)}$ with $i^{2}=-1$. It follows that

where

$$
-i=s^{2}+(1+i) \beta^{2}
$$

$$
\beta=b-(1-i) a \text {. }
$$

We must show that all solutions of (3.21) have $s^{2}=1, \beta^{2}=-1$. Following the by now 
familiar pattern, we introduce $\delta$ with

$$
\delta^{2}=-1-i \text {. }
$$

Then 2 ramifies completely in $\mathbf{Q}(i, \delta)$ and $1, i, \delta, i \delta$ is a basis for the integers. Further,

so that

$$
\operatorname{Norm}(1+i \delta)=-i \text {, }
$$

$$
\eta=1+i \delta
$$

is a unit, and it is easily checked that it is fundamental.

We have

$$
s+\beta \delta=i^{f} \eta^{\mathrm{g}}
$$

for some $f, g \in \mathbf{Z}$. By (3.21) and (3.24) $g$ is odd, say $g= \pm 1+4 n$, and so

$$
s+\beta \delta= \pm i(1 \pm i \delta) \eta^{4 n}
$$

or

$$
s+\beta \delta= \pm(1 \pm i \delta) \eta^{4 n}
$$

Now

where

$$
-\eta^{ \pm 4}=1+\theta
$$

$$
\theta=-8-8 i \pm(4-8 i) \delta \text {. }
$$

Hence (3.27) leads to a contradiction with (3.26) modulo 2.

Putting $N=|n|, 2^{r} \| n$, we have

$$
\begin{array}{rlr}
\eta^{4 n} & =(1+\theta)^{N} & \\
& \equiv 1+N \theta \quad\left(2^{r+3}\right) \\
& \equiv 1+2^{r+2} \delta \quad\left(2^{r+3}\right)
\end{array}
$$

Hence

$$
(-1)^{n}(1 \pm i \delta) \eta^{4 n} \equiv 1+2^{r+2}+2^{r+2} i+\left(2^{r+2} \pm i\right) \delta \quad\left(2^{r+3}\right) .
$$

In particular, the coefficient of $i$ is not zero, in contradiction to (3.26). Hence the only possibility is $n=0$.

\section{REFERENCES}

1. A. Baker, Transcendental number theory (Cambridge, 1975).

2. A. Baker and J. Coates, Integer points on curves of genus 1, Proc. Cambridge Philos. Soc. 67 (1970), 595-602.

3. A. Baker and H. Davenport, The equations $3 x^{2}-2=y^{2}$ and $8 x^{2}-7=z^{2}$, Quart. J. Math. Oxford Ser. 2, 20 (1969), 129-37.

4. J. W. S. Cassels, Integral points on certain elliptic curves, Proc. London Math. Soc. (3) 14A (1965), 55-57.

5. F. Ellison, W. J. Ellison, J. Pesek, C. E. Stall and D. S. Stall, The diophantine equation $y^{2}+k=x^{3}, J$. Number Theory 4 (1972), 107-117. 
6. G. Hoare, Solution and comments on 67.A and 67.B, Math. Gaz. 67 (1983), 228-230.

7. W. Ljunggren, Zur Theorie der Gleichung $x^{2}+1=D y^{4}$, Avh. Norske Vid.-Akad. Oslo, 1942 No. 5,1 .

8. L. J. Mordell, Diophantine equations (Academic Press, 1969).

9. T. Skolem, The use of $p$-adic methods in the theory of diophantine equations, Bull. Soc. Math. Belg. 7 (1955), 83-95.

D.P.M.M.S.

UNIVERSTTY OF CAMBRIDGE

16 MILl LANE

CAMBRIDGE

CB2 1SB 